\title{
THE NORM OF A SPACE CONFIGURATION
}

BY L. M. BLUMENTHAL

1. Introduction. In a recent paper, ${ }^{*}$ the writer has shown that attached to an ordered set of $2 n$ points in a plane there is the lineo-linear invariant

$$
2(N+i A)=\sum_{k=1}^{n} \bar{x}_{2 k}\left(x_{2 k-1}-x_{2 k+1}\right)=\left(\frac{1}{n}\right) \sum_{i=1}^{n-1}\left(1-\epsilon^{n-i}\right) v_{i} \bar{u}_{i},
$$

where $x_{k}(k=1,2, \cdots, 2 n)$ are points in the complex plane, $\bar{x}_{k}$ denotes the conjugate of $x_{k}, \epsilon$ is a primitive $n$th root of unity, $A$ is the area of the ordered $2 n$-gon, and the norm $N$ is defined by

$$
2 N=-\frac{1}{2}\left(\delta_{12}^{2}-\delta_{23}^{2}+\cdots+\delta_{2 n-1,2 n}^{2}-\delta_{2 n, 1}^{2}\right),
$$

with $\delta_{i j}=\left|x_{i}-x_{j}\right|$. The Lagrange resolvents $\dagger$

$$
\begin{aligned}
& v_{i}=\sum_{k=0}^{n-1} \epsilon^{i k} x_{2 k+1}, \quad \bar{u}_{i}=\sum_{k=0}^{n-1} \epsilon^{i(n-k)} \bar{x}_{2(k+1)}, \\
& \quad(i=1,2, \cdots, n-1),
\end{aligned}
$$

are absolute invariants under translations $y_{i}=x_{i}+b$, while the combinations $v_{i} \bar{u}_{i}$ are likewise invariant under rotations $y=t x_{i}$, where $t$ is a complex number with unit modulus.

This paper extends the preceding results to $S_{3}$, the theorems that are obtained holding, mutatis mutandis, for $S_{n}$. Denoting the $2 n$ points by $X_{i}(i=1,2, \cdots, 2 n)$ and calling the two sets $X_{2 i}, X_{2 i-1}(i=1,2, \cdots, n)$ the component $n$-points of the whole set, it is shown that the norm of the $2 n$-point is expressible in terms of the Lagrange resolvents of its vertices and is consequently absolutely invariant under a translation of either of its component n-points.

* Lagrange resolvents in eucilidean geometry, American Journal of Mathematics, vol. 49 (1927). pp. 511-522.

† Pascal, E., Repertorium, 2d. ed., vol. 1, p. 307. 
The vanishing of the norms of the space 6-point and 8point leads to two interesting theorems. The theorem for the latter case is seen to be the space analog of the orthologic triangle relation of Steiner.

2. Norm of Space $2 n$-Point. To express the norm of $2 n$ ordered points $X_{i} \equiv\left(x_{i}, y_{i}, z_{i}\right),(i=1,2, \cdots, 2 n)$, in terms of Lagrange resolvents, we consider first the case of the 6point. The resolvents are

$$
\begin{array}{ll}
v_{1}(x)=x_{1}+\omega x_{3}+\omega^{2} x_{5}, & u_{1}(x)=x_{2}+\omega x_{4}+\omega^{2} x_{6}, \\
v_{2}(x)=x_{1}+\omega^{2} x_{3}+\omega x_{5}, & u_{2}(x)=x_{2}+\omega^{2} x_{4}+\omega x_{6},
\end{array}
$$

with two additional sets in $y$ and $z$. If we adjoin to these sets

$$
v_{0}(x)=x_{1}+x_{3}+x_{5}, \quad u_{0}(x)=x_{2}+x_{4}+x_{6},
$$

with the accompanying forms in the other letters, we can readily solve for the coordinates in terms of $v_{i}$ and $u_{i}$ and hence show that the invariants $v_{i}, u_{i},(i=1,2)$, form a complete system.*

From the definition of $N$ we have

$$
2 N=\sum \sum_{k=1}^{3} x_{2 k}\left(x_{2 k-1}-x_{2 k+1}\right)=\sum \sum_{k=1}^{3} x_{2 k-1}\left(x_{2 k}-x_{2(3+k-1)}\right),
$$

where the summation extends over $x, y, z$ and subscripts are to be reduced mod 6, with $x_{0} \equiv x_{6}$. Substitution in the above expression yields, after some reductions,

$$
2 N=\frac{1}{1-\omega} \sum\left[v_{1}(x) \bar{u}_{1}(x)-\omega v_{2}(x) \bar{u}_{2}(x)\right]
$$

which expresses the norm of the 6-point in terms of the Lagrange resolvents of the vertices. We have, then, the following theorem.

THEOREM 1. The norm of a space 6-point is absolutely invariant under a translation of either of its component 3-points.

* The expressions $v_{0}, u_{0}$ are, of course, not invariants and will drop out of any invariant relation. 
The method illustrated above is applied to the $2 n$-point; the result being set down without further details as

$$
2 N=(1 / n) \sum \sum_{i=1}^{n-1}\left(1-\epsilon^{n-i}\right) v_{i}(x) \bar{u}_{i}(x) .
$$

THEOREM 2. The norm of a space 2n-point is absolutely invariant under translation of either of its component n-points.

3. Vanishing of the Norm. In the plane it is seen that the complex number $2(N+i A)$ is determined by an ordered set of $2 n$ points. Viewed in a somewhat different light, the expression may be regarded as a simultaneous invariant of the two component sets of $n$ points. It is easily shown that for a plane 6-point, the vanishing of the norm of the figure is a sufficient condition that lines from the vertices of one component 3-point perpendicular to corresponding joins of the other 3-point meet in a point.*

For the space 6-point we consider the three planes through the points $X_{2 i}$ perpendicular to the joins of $X_{2 i-1} X_{2 i+1}$ $(i=1,2,3)$. The equations of the planes are

$$
\begin{gathered}
\sum\left(x_{3}-x_{1}\right)\left(x-x_{2}\right)=0, \quad \sum\left(x_{5}-x_{3}\right)\left(x-x_{4}\right)=0, \\
\sum\left(x_{1}-x_{5}\right)\left(x-x_{6}\right)=0 .
\end{gathered}
$$

The determinant of the coefficients is evidently zero. Also

$$
\begin{aligned}
& K_{1}=-\left|\begin{array}{lll}
\sum x_{2}\left(x_{3}-x_{1}\right), & y_{3}-y_{1}, & z_{3}-z_{1} \\
\sum x_{4}\left(x_{5}-x_{3}\right), & y_{5}-y_{3}, & z_{5}-z_{3} \\
\sum x_{6}\left(x_{1}-x_{5}\right), & y_{1}-y_{5}, & z_{1}-z_{5}
\end{array}\right|=-2 N\left|\begin{array}{l}
y_{5}-y_{3}, z_{5}-z_{3} \\
y_{1}-y_{5}, z_{1}-z_{5}
\end{array}\right|, \\
& K_{2}=2 N\left|\begin{array}{l}
x_{5}-x_{3}, z_{5}-z_{3} \\
x_{1}-x_{5}, z_{1}-z_{5}
\end{array}\right|, K_{3}=-2 N\left|\begin{array}{ll}
x_{5}-x_{3}, & y_{5}-y_{3} \\
x_{1}-x_{5}, & y_{1}-y_{5}
\end{array}\right| .
\end{aligned}
$$

Hence the vanishing of the norm will make the expressions

* Triangles so related were called by Steiner "orthologique." He showed the relation to be reciprocal, but does not seem to have considered criteria for the existence of the relation. 
$K_{i},(i=1,2,3)$, zero, and the three planes will be coaxia .* Hence we have the following theorem.

THEOREM 3. If the norm of an ordered space 6-point vanish, planes through the vertices of one component 3-point perpendicular to the corresponding joins of the other are coaxial.

Considering the case of the space 8-point we have the following theorem.

THEOREM 4. If the norm of an ordered space 8-point vanish, the four planes through the points $X_{2 i}$ perpendicular to the joins of $X_{2 i-1} X_{2 i+1},(i=1,2,3,4)$, meet in a point.

The four planes have equations

$$
\begin{array}{ll}
\sum\left(x_{3}-x_{1}\right)\left(x-x_{2}\right)=0, & \sum\left(x_{7}-x_{5}\right)\left(x-x_{6}\right)=0, \\
\sum\left(x_{5}-x_{3}\right)\left(x-x_{4}\right)=0, & \sum\left(x_{1}-x_{7}\right)\left(x-x_{8}\right)=0 .
\end{array}
$$

They will meet in a point if and only if the determinant

$$
\Delta=\left|\begin{array}{l}
x_{3}-x_{1}, y_{3}-y_{1}, z_{3}-z_{1},-\sum x_{2}\left(x_{3}-x_{1}\right) \\
x_{5}-x_{3}, y_{5}-y_{3}, z_{5}-z_{3},-\sum x_{4}\left(x_{5}-x_{3}\right) \\
x_{7}-x_{5}, y_{7}-y_{5}, z_{7}-z_{5},-\sum x_{6}\left(x_{7}-x_{5}\right) \\
x_{1}-x_{7}, y_{1}-y_{7}, z_{1}-z_{7},-\sum x_{8}\left(x_{1}-x_{7}\right)
\end{array}\right|
$$

vanishes and not all four minors of the elements in the last column are zero. (Evidently these minors do not in general vanish.) Adding the last three rows to the first we obtain a row with three elements zero and the fourth element

that is,

$$
-\sum \sum_{k=1}^{4} x_{2 k}\left(x_{2 k+1}-x_{2 k-1}\right)
$$

which proves the theorem.

$$
\Delta=-2 N\left|\begin{array}{ccc}
x_{5}-x_{3}, & y_{5}-y_{3} & z_{5}-z_{3} \\
x_{7}-x_{5}, & \ldots & , \\
x_{1}-x_{7}, & \ldots & ,
\end{array}\right| .
$$

* The $K_{i}$ 's will be zero also if

$$
\left|\begin{array}{l}
y_{6}-y_{3}, z_{5}-z_{3} \\
y_{1}-y_{5}, z_{1}-z_{5}
\end{array}\right|=\left|\begin{array}{l}
x_{5}-x_{3}, y_{5}-y_{3} \\
x_{1}-x_{5}, y_{1}-y_{5}
\end{array}\right|=0 .
$$

The points $X_{1}, X_{3}, X_{5}$ will then be collinear and the planes will be coaxial with the line at infinity as axis. 
4. Rotation. If a rotation about the $Z$-axis of angle $\theta$ be applied to the component $n$-point $X_{2 k-1},(k=1,2, \cdots, n)$, it is seen that

$$
\begin{aligned}
& 2 N^{\prime}(x, y)=\cos \theta \cdot \sum \sum_{k=1}^{n} x_{2 k}\left(x_{2 k-1}-x_{2 k+1}\right) \\
& +\sin \theta \cdot\left[\sum_{k=1}^{n} x_{2 k}\left(y_{2 k-1}-y_{2 k+1}\right)-y_{2 k}\left(x_{2 k-1}-x_{2 k+1}\right)\right],
\end{aligned}
$$

where $N^{\prime}(x, y)$ denotes that part of the transformed norm containing only terms in $x$ and $y$ (the terms in $z$, of course, are unaltered by the rotation). Whence we may write

$$
N^{\prime}=N \cos \theta+2 N(z) \sin ^{2} \theta / 2+A \sin \theta,
$$

where $A$ is the area obtained by projecting the original $2 n$ point on the $X Y$-plane.

5. Points on a Sphere. If the points $X_{i},(i=1,2, \cdots, 2 n)$, be placed upon a sphere, we shall define the norm of the ordered $2 n$-point by

$$
2 N=\prod_{i=2,4, \cdots, 2 n}^{i=1,3, \cdots, 2 n-1} \cos X_{i} X_{i+1},
$$

where the product obtained by allowing the index to assume the values $2,4,6, \cdots, 2 n$ is to be subtracted from the product produced by letting the index take on the values $1,3,5, \cdots, 2 n-1$, and $\cos X_{i} X_{i+1}$ denotes the cosine of the arc of the great circle join of $X_{i}, X_{i+1}$.

Consider the 6 -point $X_{i},(i=1,2, \cdots, 6)$, on the sphere and through $X_{2 i}$ draw arcs of great circles perpendicular to the great circle joins of $X_{2 i-1}, X_{2 i+1}$. Suppose the arcs meet at $O$. It is readily shown that the norm of the six-point vanishes.

The Johns Hopkins University 